\title{
Pseudo-pheochromocytoma due to obstructive sleep apnea: a case report
}

\author{
Aria Jazdarehee ${ }^{D 1}$, Sawyer Huget-Penner² and Monika Pawlowska³ \\ ${ }^{1}$ Department of Medicine and Faculty of Medicine, University of British Columbia, British Columbia, Canada, ${ }^{2}$ Division \\ of Endocrinology and Metabolism, Fraser Health Authority, British Columbia, Canada, and ${ }^{3}$ Division of Endocrinology \\ and Metabolism, University of British Columbia, British Columbia, Canada
}

Correspondence should be addressed to M Pawlowska

Email

monikap@ualberta.ca

\section{Summary}

Obstructive sleep apnea (OSA) is a condition of intermittent nocturnal upper airway obstruction. OSA increases sympathetic drive which may result in clinical and biochemical features suggestive of pheochromocytoma. We present the case of a 65-year-old male with a 2.9-cm left adrenal incidentaloma on CT, hypertension, symptoms of headache, anxiety and diaphoresis, and persistently elevated 24-h urine norepinephrine (initially $818 \mathrm{nmol} /$ day (89-470)) and normetanephrine (initially $11.2 \mu \mathrm{mol} /$ day (0.6-2.7)). He was started on prazosin and underwent left adrenalectomy. Pathology revealed an adrenal corticoadenoma with no evidence of pheochromocytoma. Over the next 2 years, urine norepinephrine and normetanephrine remained significantly elevated with no MIBG avid disease. Years later, he was diagnosed with severe OSA and treated with continuous positive airway pressure. Urine testing done once OSA was well controlled revealed complete normalization of urine norepinephrine and normetanephrine with substantial symptom improvement. It was concluded that the patient never had a pheochromocytoma but rather an adrenal adenoma with biochemistry and symptoms suggestive of pheochromocytoma due to untreated severe OSA. Pseudo-pheochromocytoma is a rare presentation of OSA and should be considered on the differential of elevated urine catecholamines and metanephrines in the right clinical setting.

\section{Learning points:}

- Obstructive sleep apnea (OSA) is a common condition among adults.

- OSA may rarely present as pseudo-pheochromocytoma with symptoms of pallor, palpitations, perspiration, headache, or anxiety.

- OSA should be considered on the differential of elevated urine catecholamines and metanephrines, especially in patients with negative metaiodobenzylguanidine (MIBG) scan results.

\section{Background}

Adrenal incidentalomas are a common finding in patients undergoing abdominal and pelvic CT scans and require functional assessment once identified. Pheochromocytoma is diagnosed in $5 \%$ of functional adrenal incidentalomas and must be excluded given its high morbidity and mortality $(1,2)$.

Pheochromocytomas and paragangliomas are catecholamine-secreting tumors derived from the adrenal medulla and nerve cell paraganglia, respectively (3). Classical symptoms include headache, palpitations, diaphoresis, hypertension, and pallor. Life-threatening complications including hypertensive crisis, catecholamine-induced cardiomyopathy, and multi-organ dysfunction may occur due to prolonged catecholamine release. Diagnosis can be challenging, as clinical symptoms vary and localization of the 
catecholamine-secreting tumor may be difficult. Diagnosis is made based on elevated catecholamines and metanephrines in conjunction with localization studies. Laparoscopic adrenalectomy is the mainstay of treatment for pheochromocytoma (4).

Pseudo-pheochromocytoma is a disorder that presents similarly to pheochromocytoma with paroxysmal hypertension, palpitations, diaphoresis, and chest pain (5). However, unlike pheochromocytomas and paragangliomas, pseudo-pheochromocytomas present in the absence of catecholamine-secreting tumors. Pseudopheochromocytoma is a diagnosis of exclusion, and commonly associated conditions include obstructive sleep apnea (OSA), physiologic stress, antipsychotic drugs, antiParkinson drugs, tricyclic antidepressants, and cocaine use.

\section{Case presentation}

A 65-year-old male patient initially presented to endocrinology in February 2010 for assessment of a 2.9$\mathrm{cm}$ left adrenal incidentaloma that was found on CT abdomen that was done for right upper quadrant pain and cholelithiasis (Fig. 1). The Hounsfield units ranged from -7 to 17 . He reported a several month history of increased headaches, nocturnal diaphoresis, and increased anxiety.

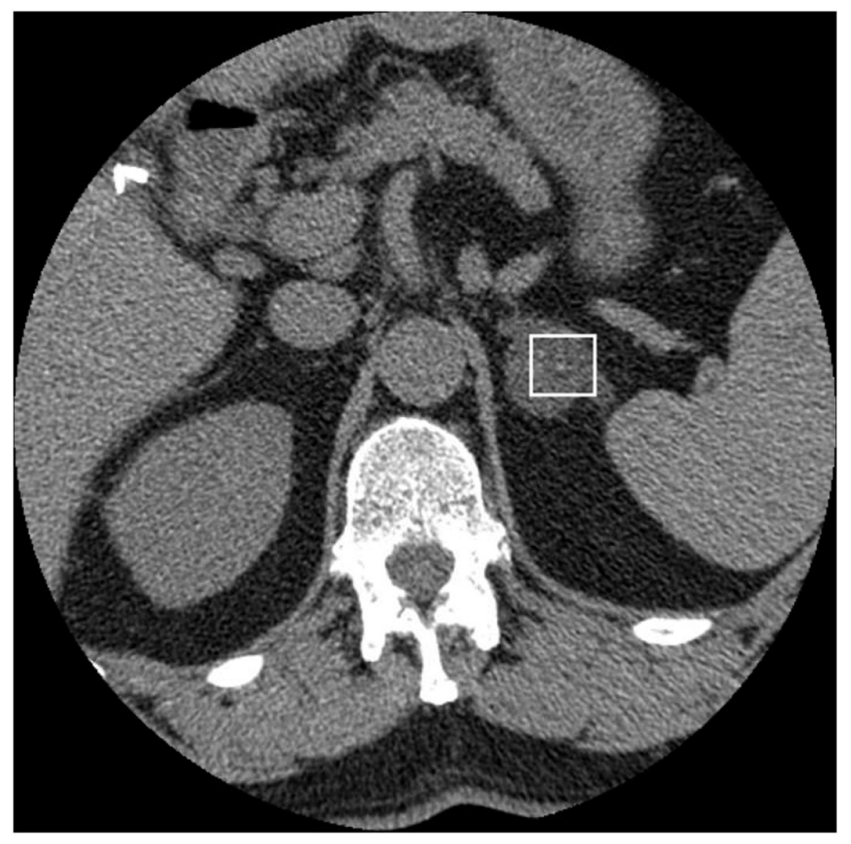

\section{Figure 1}

Left adrenal incidentaloma noted on enhanced CT. The mass was a well-circumscribed hypodense left adrenal nodule measuring $2.9 \mathrm{~cm}$. The Hounsfield units ranged from -7 to 17 . Pathological analysis following left adrenalectomy demonstrated an adrenal cortical adenoma with negative immunohistochemical staining for pheochromocytoma.
His initial blood pressure in the office was elevated at $160 / 98 \mathrm{mmHg}$ with no prior history of hypertension.

\section{Investigation}

His endocrine workup revealed elevated 24-h urine norepinephrine of $818 \mathrm{nmol} /$ day (89-470) and normetanephrine of $11.2 \mu \mathrm{mol} /$ day $(0.6-2.7)$, and repeat testing showed persistent elevation in normetanephrines at $9.9 \mathrm{nmol} /$ day (0.6-2.7) (Table 1). Measurements of urine norepinephrine and normetanephrine were completed using high-performance liquid chromatography and electrochemical detection. He was on no interfering medications at that time. Given the adrenal incidentaloma, workup for primary hypoaldosteronism and Cushing's syndrome was done, with normal 24-h urine aldosterone and 24-h urinary free cortisol.

\section{Treatment}

Given his symptoms, imaging, and investigations, he was diagnosed with a pheochromocytoma, started on alpha-blockade with prazosin, and referred for surgery. His prazosin was slowly titrated to $2 \mathrm{mg}$ twice-a-day preoperatively, and he had a left adrenalectomy in July 2010.

Pathology revealed an adrenal corticoadenoma with no evidence of pheochromocytoma. There was a persistent and significant elevation in his 24-h urine norepinephrine and normetanephrine over the next 2 years (Table 1). Given his negative pathology, a paraganglioma was suspected. However, there was no evidence of an extraadrenal source, with three MIBG scans showing no evidence of MIBG avid disease and normal CT scans of the head, spine, chest, and abdomen. His blood pressure improved without initiation of antihypertensives, but he continued to have persistent headaches, anxiety, and diaphoresis.

\section{Outcome and follow-up}

In May 2012, he was diagnosed as having OSA and was initiated on treatment with nocturnal continuous positive airway pressure (CPAP). After his OSA was treated, repeat urine testing showed complete normalization of urine norepinephrine and metanephrines with substantial symptom improvement (Table 2).

It was concluded that the patient never had a pheochromocytoma and that he had a non-functioning adrenal adenoma with biochemistry and symptoms 
Table 1 24-h urine catecholamine and metanephrine results prior to OSA treatment. The patient underwent a left adrenalectomy in July 2010.

\begin{tabular}{l}
\hline Date \\
\hline Metanephrine \\
Normetanephrine \\
Epinephrine \\
Norepinephrine \\
Dopamine \\
Creatinine \\
\hline
\end{tabular}

\begin{tabular}{cccc}
\hline May 2010 & & June 2010 \\
\cline { 1 - 1 } 0.5 & & 0.5 \\
$\mathbf{1 1 . 2}$ & & $\mathbf{9 . 9}$ \\
$<28$ & & $<31$ \\
$\mathbf{8 1 8}$ & & 426 \\
3.9 & & 2.1 \\
$\mathbf{2 4 . 1}$ & & 9.9 \\
\hline
\end{tabular}

\begin{tabular}{c}
\hline Aug 2010 \\
\hline 19 \\
$<13$ \\
190 \\
1 \\
\hline
\end{tabular}

\begin{tabular}{c}
\hline Aug 2011* \\
\hline$<0.4$ \\
$\mathbf{8 . 6}$ \\
$<18$ \\
$\mathbf{7 5 2}$ \\
1.6 \\
12.8 \\
\hline
\end{tabular}

\begin{tabular}{c}
\hline April 2012** \\
\hline$<0.4$ \\
$\mathbf{1 1 . 1}$ \\
29 \\
$\mathbf{1 0 0 7}$ \\
2.2 \\
16.7 \\
\hline
\end{tabular}

\begin{tabular}{c}
\hline April 2012*** \\
\hline 0.23 \\
$\mathbf{5 . 3}$ \\
$<24$ \\
$\mathbf{5 7 4}$ \\
1.6 \\
12.3 \\
\hline
\end{tabular}

\begin{tabular}{c}
\hline Oct $\mathbf{2 0 1 2}$ \\
\hline 0.4 \\
$\mathbf{1 0 . 8}$ \\
$<24$ \\
$\mathbf{7 6 6}$ \\
2.4 \\
16.9 \\
\hline
\end{tabular}

\begin{tabular}{c}
\hline Dec 2013 \\
\hline$<0.4$ \\
$\mathbf{5 . 1}$ \\
$<20$ \\
$\mathbf{4 7 1}$ \\
2.3 \\
11.7 \\
\hline
\end{tabular}

\begin{tabular}{l}
\multicolumn{1}{c}{ N Range } \\
\hline$<0.20 \mu \mathrm{mol} / \mathrm{day}$ \\
$<0.20 \mu \mathrm{mol} / \mathrm{day}$ \\
$<160 \mathrm{nmol} / \mathrm{day}$ \\
$89-470 \mathrm{nmol} / \mathrm{day}$ \\
$0.4-3.3 \mu \mathrm{mol} / \mathrm{day}$ \\
$7-18 \mathrm{nmol} / \mathrm{day}$ \\
\hline
\end{tabular}

* On amitriptyline for headaches; ** Off amitriptyline, on beta blocker; *** Off amitriptyline and beta blocker.

Bold values represent lab results outside the normal limits.

suggestive of pheochromocytoma due to untreated severe OSA, which resolved with CPAP therapy.

\section{Discussion}

OSA is a common condition that affects up to $9-38 \%$ of adults (6). It is diagnosed based on overnight oximetry testing or with polysomnography. Recurrent hypoxia with repeated sudden arousal triggers a surge of sympathetic nervous activity which can result in elevated urine catecholamines and metanephrines and a clinical picture of pheochromocytoma $(7,8)$. OSA is a known risk factor for cardiovascular disease, stroke, and hypertension. This risk may be due to the elevated catecholamines and metanephrines from recurrent hypoxia. Treatment is with CPAP, which decreases sympathetic tone by preventing upper airway closure and stopping recurrent hypoxia (9).

OSA rarely presents as a pseudo-pheochromocytoma with elevated catecholamines and metanephrines along with symptoms of pallor, palpitations, perspiration, headache or anxiety, and has only been documented in case reports. Similar to our case, previous reports note resolution of clinical symptoms and normalization of

Table 2 24-h urine catecholamine and metanephrine results after OSA treatment. The patient underwent a left adrenalectomy in July 2010.

\begin{tabular}{|c|c|c|c|c|}
\hline Date & $\begin{array}{l}\text { Jan } \\
2017\end{array}$ & $\begin{array}{c}\text { March } \\
3,2017^{*}\end{array}$ & $\begin{array}{c}\text { March } \\
28,2017\end{array}$ & N Range \\
\hline Metanephrine & 0.04 & 0.08 & 0.04 & $<0.20 \mu \mathrm{mol} / \mathrm{day}$ \\
\hline Normetanephrine & 0.45 & 0.70 & 0.37 & $<0.20 \mu \mathrm{mol} / \mathrm{day}$ \\
\hline Epinephrine & 26 & 39 & 19 & $<90$ nmol/day \\
\hline Norepinephrine & 396 & 774 & 422 & $<650 \mathrm{nmol} /$ day \\
\hline Dopamine & 2676 & 3715 & 1389 & 400-3300 nmol/day \\
\hline Creatinine & 19.5 & 35.8 & 14.3 & 7-18 nmol/day \\
\hline
\end{tabular}

*Probable over collection of urine.

Bold values represent lab results outside the normal limits. catecholamines and metanephrines with proper treatment of OSA with CPAP (7).

When a patient presents with suspicion for pheochromocytoma but imaging does not identify a mass, a pseudo-pheochromocytoma should be considered. If an adrenal mass is present, biochemical features may be used to support the diagnosis of pheochromocytoma. Elevations in both metanephrine and normetanephrine levels are most consistent with adrenal localization (10). Isolated normetanephrine elevation without metanephrine elevation, as in our case, may point away from a diagnosis of pheochromocytoma, but adrenal or extra-adrenal localization with likely $V H L$ or $S D H x$ mutations still needs to be considered (10). In addition to OSA, physiologic stress, antipsychotic drugs, anti-Parkinson drugs, tricyclic antidepressants, and cocaine use are on the differential diagnosis of a pseudo-pheochromocytoma (5).

It is important to properly diagnose pseudopheochromocytoma due to OSA to avoid unnecessary surgery along with its potential risks and to decrease the associated comorbidities of OSA. Appropriate treatment of OSA with CPAP should normalize urinary catecholamines andmetanephrinesand help resolveassociated symptomsin the setting of OSA-associated pseudo-pheochromocytoma.

Declaration of interest

The authors declare that there is no conflict of interest that could be perceived as prejudicing the impartiality of the research reported.

\section{Funding}

This study did not receive any specific grant from any funding agency in the public, commercial or not-for-profit sector.

Patient consent

Written informed consent has been obtained from the patient for publication of the submitted article. This has been documented in the patient's medical records. 


\section{Author contribution statement}

A J, S H P, and M P drafted the manuscript and approved the final version. S H P and M P were directly involved in the patient's care.

\section{References}

1 Gardner DG \& Shoback D. Greenspan's basic and clinical endocrinology. In Greenspan's Basic and Clinical Endocrinology [Internet], 10th ed. New York, NY: McGraw-Hill Education, 2017. (available at: accessmedicine.mhmedical.com/content. aspx?aid=1144813132)

2 Gendy R \& Rashid P. RACGP - incidental adrenal masses - a primary care approach [Internet]. [cited 6 Sept 2021]. (available at: (https:// www.racgp.org.au/afp/2017/june/incidental-adrenal-masses-aprimary-care-approach/)

3 Neumann HPH, Young WF \& Eng C. Pheochromocytoma and paraganglioma. New England Journal of Medicine 2019381 552-565. (https://doi.org/10.1056/NEJMra1806651)

4 Lenders JWM, Duh QY, Eisenhofer G, Gimenez-Roqueplo AP, Grebe SKG, Murad MH, Naruse M, Pacak K, Young WF \& Endocrine Society. Pheochromocytoma and paraganglioma: an Endocrine Society clinical practice guideline. Journal of Clinical Endocrinology and Metabolism 201499 1915-1942. (https://doi.org/10.1210/jc.2014-1498)
5 Mamilla D, Gonzales MK, Esler MD \& Pacak K.

Pseudopheochromocytoma. Endocrinology and Metabolism Clinics of North America 201948 751-764. (https://doi.org/10.1016/j. ecl.2019.08.004)

6 Senaratna CV, Perret JL, Lodge CJ, Lowe AJ, Campbell BE, Matheson MC, Hamilton GS \& Dharmage SC. Prevalence of obstructive sleep apnea in the general population: a systematic review. Sleep Medicine Reviews 201734 70-81. (https://doi.org/10.1016/j. smrv.2016.07.002)

7 Hoy LJ, Emery M, Wedzicha JA, Davison AG, Chew SL, Monson JP \& Metcalfe KA. Obstructive sleep apnea presenting as pseudopheochromocytoma: a case report. Journal of Clinical Endocrinology and Metabolism 200489 2033-2038. (https://doi. org/10.1210/jc.2003-031348)

8 Weeks AC, Kimple ME \& Davis DB. The importance of exclusion of obstructive sleep apnea during screening for adrenal adenoma and diagnosis of pheochromocytoma. Journal of Investigative Medicine High Impact Case Reports 201532324709615607062 . (https://doi. org/10.1177/2324709615607062)

9 Cheezum MK \& Lettieri CJ. Obstructive sleep apnea presenting as pseudopheochromocytoma. Journal of Clinical Sleep Medicine 20106 190-191. (https://doi.org/10.5664/jcsm.27770)

10 Eisenhofer G \& Peitzsch M. Laboratory evaluation of pheochromocytoma and paraganglioma. Clinical Chemistry 201460 1486-1499. (https://doi.org/10.1373/clinchem.2014.224832)

Received in final form 17 December 2021

Accepted 2 February 2022 